\title{
Efecto de la concentración del silano MTMO sobre la protección contra la corrosión de acero electrocincado
}

\author{
Effect of MTMO silane concentration on \\ electrogalvanized steel in the protection \\ against corrosion
}

\author{
Pablo Ricardo Seré ${ }^{1,2}$, Walter Alfredo Egli ${ }^{1}$, Cecilia Inés Elsner ${ }^{1,2}$, \\ Alejandro Ramón Di Sarli ${ }^{1}$, Cecilia Deyá ${ }^{1,2}$
}

\footnotetext{
${ }^{1}$ Grupo de Análisis Electroquímico de Pinturas y Recubrimientos, Centro de Investigación y Desarrollo en Tecnología de Pinturas (CIDEPINT), CICPBA-CONICET, Av. 52 s/No, CP1900, La Plata, Buenos Aires, Argentina. e-mail: anelpire@ cidepint.gov.ar

2 .Facultad de Ingeniería, Universidad Nacional de La Plata, Av 1 esq 47, CP 1900, La Plata, Buenos Aires, Argentina. e-mail: cielsner@ing.unlp.edu.ar
}

\section{RESUMEN}

Los pretratamientos superficiales a base de silanos son una de las alternativas más prominentes para reemplazar a los cromatos como protectores temporarios de sustratos metálicos expuestos a la acción de ambientes agresivos. El mecanismo de protección de los silanos, por efecto barrera, hace que características como porosidad, espesor y homogeneidad de la película tengan una marcada influencia sobre su eficiencia. En el presente trabajo se analizó la influencia que la concentración y cantidad de capas de mercaptopropiltrimetoxisilano (MTMO) aplicadas a chapas de acero electrocincado ejercen sobre el comportamiento de estas últimas frente a la corrosión. Previo a la aplicación del silano, las muestras fueron desengrasadas por ultrasonido sumergiéndolas en tolueno durante $2 \mathrm{~min}$ a $40^{\circ} \mathrm{C}$. La superficie de las muestras fue activada sumergiéndolas en una solución de $\mathrm{NaOH}$ al $10 \%$ p/v y aplicando una densidad de corriente catódica de $0,12 \mathrm{~A} / \mathrm{cm}^{2}$, la temperatura se controló a $40{ }^{\circ} \mathrm{C}$. La solución de MTMO se preparó añadiendo 2 ó 4\% v/v de MTMO a una solución de agua destilada/metanol (3:2 v/v, de pH 4 ajustado con ácido acético) y se hidrolizó durante $60 \mathrm{~min}$; a algunas muestras se les aplicó una y a otras dos capas de MTMO. Posteriormente se curaron a $80{ }^{\circ} \mathrm{C}$ durante 10 min. La porosidad se evaluó por voltametría cíclica y por reacción al sulfato de cobre, la morfología y espesor mediante SEM-EDS, y el comportamiento frente a la corrosión mediante la obtención de curvas de polarización, espectrometría de impedancia electroquímica y exponiendo las muestras en cámara de humedad y temperatura controladas. De los resultados obtenidos puede inferirse que la capacidad protectora aportada por dos capas de MTMO es mayor que la debida a un aumento de su concentración. Además, estas variables de proceso afectan tanto la morfología como el espesor de la película.

Palabras claves: Silanos, acero electrocincado, protección anticorrosiva, MTMO

\section{ABSTRACT}

Superficial pretreatments based on silanes are one of the prominent alternatives to replace chromates as temporary protectors from the action of aggressive environments to metallic substrates. The barrier effect afforded by the silane film makes porosity, thickness and film homogeneity important issues in the protective effect. In the present work, the influence of the concentration and amount of layers of mercaptopropyltrimethoxysilane (MTMO) applied on electrogalvanized steel on its corrosion behavior was evaluated. Before silane application the substrate was degreased by immersion in toluene for $2 \mathrm{~min}$ at $40{ }^{\circ} \mathrm{C}$. The surface sample was activated by immersion in $\mathrm{NaOH} 10 \% \mathrm{p} / \mathrm{v}$ and applying a cathodic current density of $0,12 \mathrm{~A} / \mathrm{cm}^{2}$, the temperature was controlled at $40{ }^{\circ} \mathrm{C}$. MTMO was prepared adding 2 or $4 \% \mathrm{v} / \mathrm{v}$ of MTMO to a distilled water/methanol $(3: 2 \mathrm{v} / \mathrm{v}, \mathrm{pH}$ adjusted to 4 with acetic acid), and hydrolyzed for $60 \mathrm{~min}$; some samples were coated with 1 layer of MTMO and others with 2 layers. All the coatings were cured at $80{ }^{\circ} \mathrm{C}$ for 10 min. The coating's porosity was evaluated by cyclic voltammetry and reaction to copper sulphate, its thickness and 
morphology by SEM-EDS and the corrosion behavior by polarization curves, electrochemical impedance spectroscopy and exposure into a temperature and humidity controlled-chamber. The results showed that the protective capacity afforded by 2 layers of MTMO is higher than that obtained by raising the silane concentration. Besides, the variables under study affect the morphology and the thickness of the protective layer.

Keywords: Silanes, electrogalvanized steel, anticorrosive protection, MTMO.

\section{INTRODUCCIÓN}

Desde hace muchos años el cincado del acero ha demostrado ser un eficiente y económico método de protección contra la corrosión atmosférica [1,2]. Para prevenir la oxidación del cinc durante el almacenamiento de productos elaborados con acero galvanizado se utilizan pretratamientos a base de cromo hexavalente, este método es muy eficiente y económico. Sin embargo, el efecto de las sales de $\mathrm{Cr}^{+6}$ sobre el medio ambiente y la salud de las personas ha hecho que cada vez haya más limitaciones para su uso [3]. Por esta razón desde hace algunos años se están investigando distintas alternativas, dentro de las cuales, los silanos organofuncionales aparecen como una de las más interesantes para proteger sustratos metálicos ya que tienen muy bajo impacto ambiental, no son nocivos para la salud de las personas y tienen buena compatibilidad con un rango importante de interfaces tanto orgánicas cómo inorgánicas [4-7]. Los silanos organofuncionales poseen en un extremo de la molécula un grupo hidrolizable y en el otro un grupo orgánico funcional. El grupo alcoxido produce silanol en la hidrólisis, que luego se enlaza a la superficie del metal por condensación a través del grupo hidroxilo presente en la misma, formando el enlace Si-O-Metal, que dependiendo de la naturaleza del metal, puede ser muy estable. El otro terminal de la cadena consiste en grupos orgánicos específicos, como por ejemplo los grupos epoxi, amino, mercapto los cuales pueden interactuar con distintos compuestos orgánicos, por ejemplo una matriz de pintura [8-10]. Los silanos organofuncionales protegen al sustrato metálico de la corrosión sólo por efecto barrera ya que son electroquímicamente inertes. Por esta razón el poder protector que brindan es muy dependiente de las características de la película que forman, por ejemplo: espesor, uniformidad, cantidad de defectos como porosidad o fisuras son variables que afectan la vida útil del sistema metal/pretratamiento [11,12]. En el presente trabajo se estudió el efecto de la concentración y las condiciones de aplicación (una o dos capas) de MTMO sobre el efecto protector que presenta este silano cuando es aplicado en acero electrocincado; la morfología del sustrato y del recubrimiento de MTMO se caracterizó por SEM y la composición por EDS. La porosidad de la película por voltametría cíclica (VC) y por reacción al sulfato de cobre. El comportamiento frente a la corrosión mediante técnicas electroquímicas como espectroscopia de impedancia electroquímica (EIE), curvas de polarización (CP) y exposición en cámara de humedad $(\mathrm{CH})$.

\section{MATERIALES Y MÉTODOS}

Se prepararon muestras de 7,5 x 10,0 x 0,07 cm de acero electrocincado comercial de calidad automotriz, el acero base utilizado fue grado SAE 1004 y el espesor del recubrimiento de cinc de $7 \mu \mathrm{m}$. Todas las muestras fueron sometidas a limpieza por ultrasonido en tolueno a $40^{\circ} \mathrm{C}$ durante 7 minutos, posteriormente su superficie fue activada electroquímicamente en una solución de $\mathrm{NaOH}$ al $10 \%$ p/v aplicando una densidad de corriente catódica de $0,12 \mathrm{~A} / \mathrm{cm}^{2}$ durante $20 \mathrm{~s}$, la temperatura se controló a $40^{\circ} \mathrm{C}$. La solución de MTMO se preparó añadiendo 2\% o 4\% v/v de MTMO a una solución de agua destilada/metanol (3:2 v/v) y el pH se ajustó a 4 con ácido acético, el tiempo de inmersión fue de $1 \mathrm{~min}$ y el curado se realizó a $80^{\circ} \mathrm{C}$ durante 10 min [13]. En algunos casos, luego de la inmersión en MTMO las muestras se secaron con chorro de aire caliente y se les realizó una segunda inmersión; el curado de la película se realizó en las condiciones ya mencionadas.

Luego del curado, las películas de silano fueron observadas mediante microscopía electrónica de barrido (SEM) utilizando un microscopio Quanta 200® FEI con detector de electrones Apollo 40, la composición de la película se determinó mediante espectroscopia de dispersión de rayos X (EDS) utilizando un detector EDAX. Para evaluar la porosidad y estimar el espesor de las películas de conversión, se aplicó sobre las muestras sulfato de cobre y además, se realizaron medidas de Voltametría Cíclica. Para el ensayo de solución de sulfato de cobre se utilizó una solución de 36 g de $\mathrm{CuSO}_{4} .5 \mathrm{H}_{2} \mathrm{O}$ en $100 \mathrm{~mL}$ de $\mathrm{H}_{2} \mathrm{O}$ destilada, la solución se saturó en $\mathrm{Cu}(\mathrm{OH})_{2}$ para neutralizarla. A las muestras se le aplicó la solución durante $10 \mathrm{~s}$ y luego se enjuagaron en agua destilada. El $\mathrm{Zn}$ expuesto reacciona con el $\mathrm{Cu}^{2+}$ dando $\mathrm{Cu}^{\circ}$ que se deposita sobre la superficie revelando los poros. De esta manera por observación visual y microscopía óptica se puede evaluar el grado de porosidad de las películas de conversión en relación con la cantidad de sitios activos de zinc en los cuales se depositó el cobre. Por otro lado, la voltametría cíclica se realizó en una solución de borato aireado (35 g/L de $\mathrm{H}_{3} \mathrm{BO}_{3}$ y $40 \mathrm{~g} / \mathrm{L}$ de $\mathrm{Na}_{2} \mathrm{~B}_{4} \mathrm{O}_{7} .10 \mathrm{H}_{2} \mathrm{O}$ ) [14-16]. El barrido de potencial se realizó entre $-1500 \mathrm{mV}$ y $+500 \mathrm{mV}$ vs Electrodo de Calomel Saturado (ECS), con una velocidad de barrido de $100 \mathrm{mV} / \mathrm{s}$ a temperatura 
ambiente. En este electrolito, la VC para electrodos de zinc muestra un pico de disolución anódico seguido por una región pasiva. La integración de este pico da la carga anódica proporcional al área activa de cinc.

Se realizaron ensayos de polarización lineal de los cuales se obtuvieron curvas de polarización en una solución $0,05 \mathrm{~mol} / \mathrm{L}$ de $\mathrm{NaCl}$ a $22 \pm 1^{\circ} \mathrm{C}$. La velocidad y amplitud de barrido del potencial fueron de $0,166 \mathrm{mV} / \mathrm{s}$ y $\pm 50 \mathrm{mV}$ a partir del potencial de circuito abierto (PCA), respectivamente. El área del electrodo de trabajo fue $1 \mathrm{~cm}^{2}$. Una malla de platino de gran área se utilizó como contraelectrodo y un ECS como referencia. La densidad de corriente de corrosión $\left(\mathrm{j}_{\text {corr }}\right)$ fue obtenida por regresión lineal del gráfico E vs. $\log (\mathrm{j})$ en un rango de potencial $\pm 10 \mathrm{mV}$ con respecto al PCA [17]. Antes de cada barrido, los electrodos permanecieron sumergidos en el electrolito hasta obtener lecturas estables del potencial de circuito abierto. Las voltametrías cíclicas y las curvas de polarización se obtuvieron con un potenciostato/galvanostato PAR 273A controlado por el software CorrWear®. Para realizar las medidas de EIE se utilizó una celda constituida por un tubo de acrílico que fue pegado sobre cada sustrato con un adhesivo siliconado, dejando un área expuesta de $15,9 \mathrm{~cm}^{2}$. Como electrolito se utilizó una solución $0,05 \mathrm{M} \mathrm{NaCl}$, como electrodo de referencia un ECS y como contraelectrodo una malla de platino de gran área, las medidas se realizaron a $22 \pm 1{ }^{\circ} \mathrm{C}$. Las medidas se realizaron en el PCA con una amplitud de la señal sinusoidal de $10 \mathrm{mV}$ pico a pico en el rango de frecuencia $65.10^{3} \mathrm{~Hz}<\mathrm{f}<1.10^{-2} \mathrm{~Hz}$. Las determinaciones se realizaron con un FRA Solartron ${ }^{\circledR} 1255$ acoplado con una EI Solarton ${ }^{\circledR}$ 1286, ambos controlados por el programa Zplot ${ }^{\circledR}$. En la Tabla 1 se detalla la identificación de las muestras.

Además, un set de muestras se colocaron en una cámara de temperatura y humedad controladas (ASTM D-2247) y se evaluó el comportamiento protector visualmente luego de 264 horas de exposición.

Tabla 1: Identificación de las muestras ensayadas.

\begin{tabular}{c|c}
\hline IDENTIFICACIÓN DE LAS MUESTRAS & DESCRIPCIÓN \\
\hline $2 \mathrm{M} 1 \mathrm{C}$ & MTMO al 2\% aplicado en una capa \\
\hline $2 \mathrm{M} 2 \mathrm{C}$ & MTMO al 2\% aplicado en dos capas \\
\hline $4 \mathrm{M} 1 \mathrm{C}$ & MTMO al 4\% aplicado en una capa \\
\hline $4 \mathrm{M} 2 \mathrm{C}$ & MTMO al 4\% aplicado en dos capas \\
\hline AEC & Acero electrocincado sin recubrimiento de MTMO \\
\hline
\end{tabular}

\section{RESULTADOS Y DISCUSIÓN}

\subsection{Morfología, composición y porosidad de los recubrimientos de MTMO}

Los resultados de EDS se muestran en la Figura 1 donde puede observarse que la cantidad de Si y S aumenta con la concentración de MTMO y con el número de capas aplicadas. Si bien la penetración del haz de electrones es mucho mayor que el espesor del recubrimiento de MTMO y la contribución de la película en estudio es un porcentaje relativamente bajo de la señal es viable cuantificar todos los elementos presentes en el recubrimiento. Por otra parte, como el sustrato es el mismo, es posible comparar la composición de los distintos recubrimientos generados. La tendencia mostró que la concentración atómica de Si y S aumenta con el aumento de la concentración de silano en solución, lo que puede interpretarse como que el espesor del recubrimiento aumenta también conforme se incrementa la concentración de silano y, por ende, sería válido considerar que el aumento porcentual de Si y S es proporcional al aumento de espesor. Hansal et al. [18] determinaron por elipsometría espesores de películas de MTMO aplicadas sobre acero electrocincado a distintas concentraciones de silano en solución y encontraron que la relación entre el aumento de concentración y el espesor de recubrimiento no seguía una ley lineal. Del análisis de los resultados mostrados en la Figura 1 pudo establecerse que el aumento de Si y S, determinado por EDS, al aumentar la concentración de MTMO en solución presenta una relación semejante a la observada por Hansal et al. Esto es indicativo que sería viable correlacionar el contenido de Si y S con el cambio de espesor cuando se comparen películas del mismo silano aplicadas sobre el mismo sustrato. Partiendo de esta premisa podría inferirse que el espesor de los recubrimientos $2 \mathrm{M} 2 \mathrm{C}$ y $4 \mathrm{M} 1 \mathrm{C}$ son similares y que el aumento de espesor de película que provocó la segunda inmersión en la solución de MTMO es del orden del 10\% para la menor concentración de MTMO y del 15\% para la de mayor concentración.

En las micrografías SEM mostradas en las Figuras $\mathbf{2 b}$ y $\mathbf{3 b}$ se observa que para ambas concentracio- 
nes de MTMO y doble inmersión la superficie de cinc se encuentra totalmente cubierta por la película de MTMO. Con respecto a la morfología de los recubrimientos, sólo el correspondiente a la muestra $2 \mathrm{M} 1 \mathrm{C}$ no resultó totalmente homogéneo (Figura 3a); en el resto de los casos el recubrimiento cubrió totalmente la superficie de cinc y no se evidenciaron las típicas fisuras que habitualmente se presentan a consecuencia de las tensiones internas que se generan durante el curado.

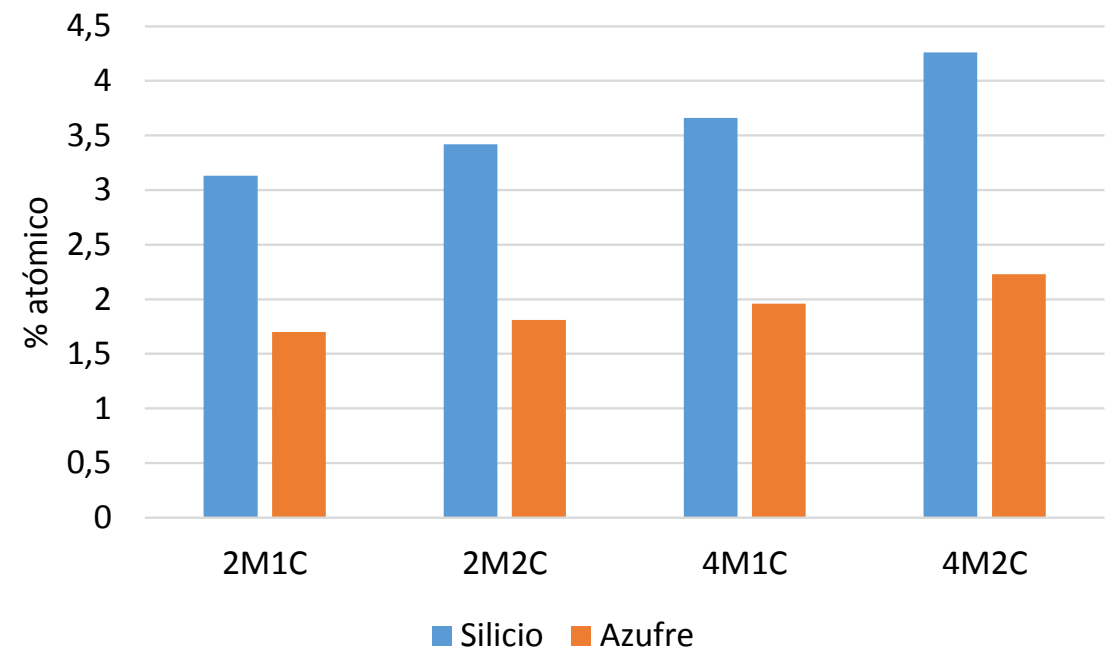

Figura 1: Concentración de Silicio y Azufre en el recubrimiento determinada por EDS.
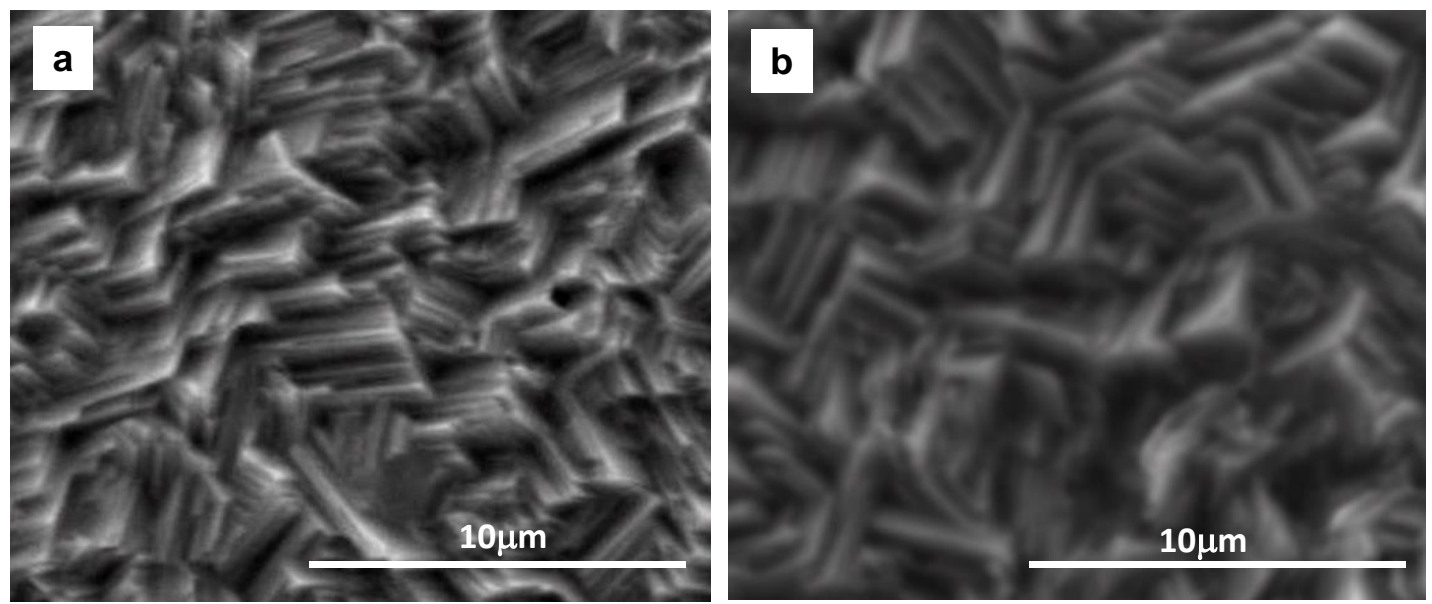

Figura 2: Fotografías SEM; a) muestra AEC, b) muestra 4M2C.
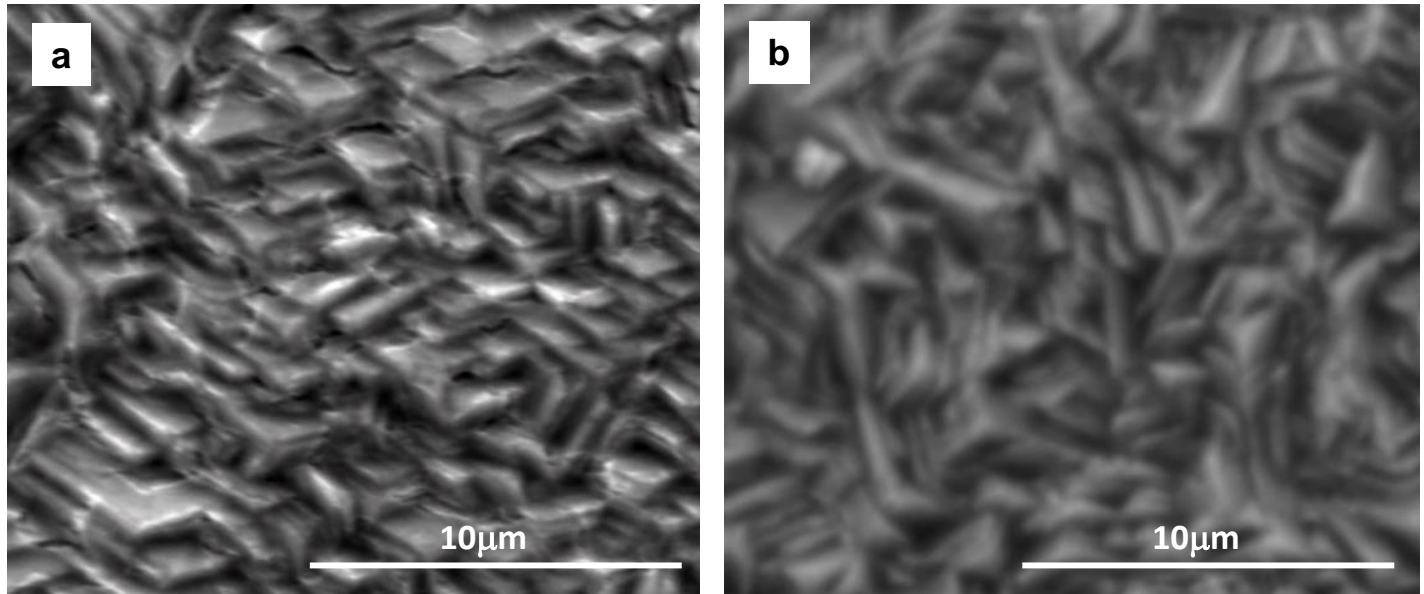

Figura 3: Fotografías SEM; a) muestra 2M1C, b) muestra 2M2C. 
La Figura 4 muestra que la respuesta de la voltamperometría correspondiente a la muestra AEC presenta un pico anódico "A" a aproximadamente $-1,0 \mathrm{~V} /(\mathrm{ECS})$ correspondiente a la oxidación del cinc, seguido por una región pasiva y un pico catódico "B" corresponde a la reducción del óxido o hidróxido de cinc [14]. El pico anódico corresponde a la formación de una película de $\mathrm{ZnO}$ o $\mathrm{Zn}(\mathrm{OH})_{2}$ que bloquea la superficie activa promoviendo el proceso de pasivación. Dado que el mecanismo de protección del MTMO es por barrera, la presencia de la película traería como consecuencia el bloqueo de los sitios activos de la superficie del sustrato y, por ende, si la película fuese homogénea y contínua los picos A y B deberían desdibujarse. Si esto no ocurre, la consecuencia lógica es aceptar que la película aplicada no resultó homogénea ni continua, que la barrera generada no es totalmente efectiva y que parte de la superficie del cinc permanece sin protección. El área bajo el pico " $\mathrm{A}$ " será proporcional a la superficie expuesta de cinc y, consecuentemente, a la porosidad de la película de MTMO [15].

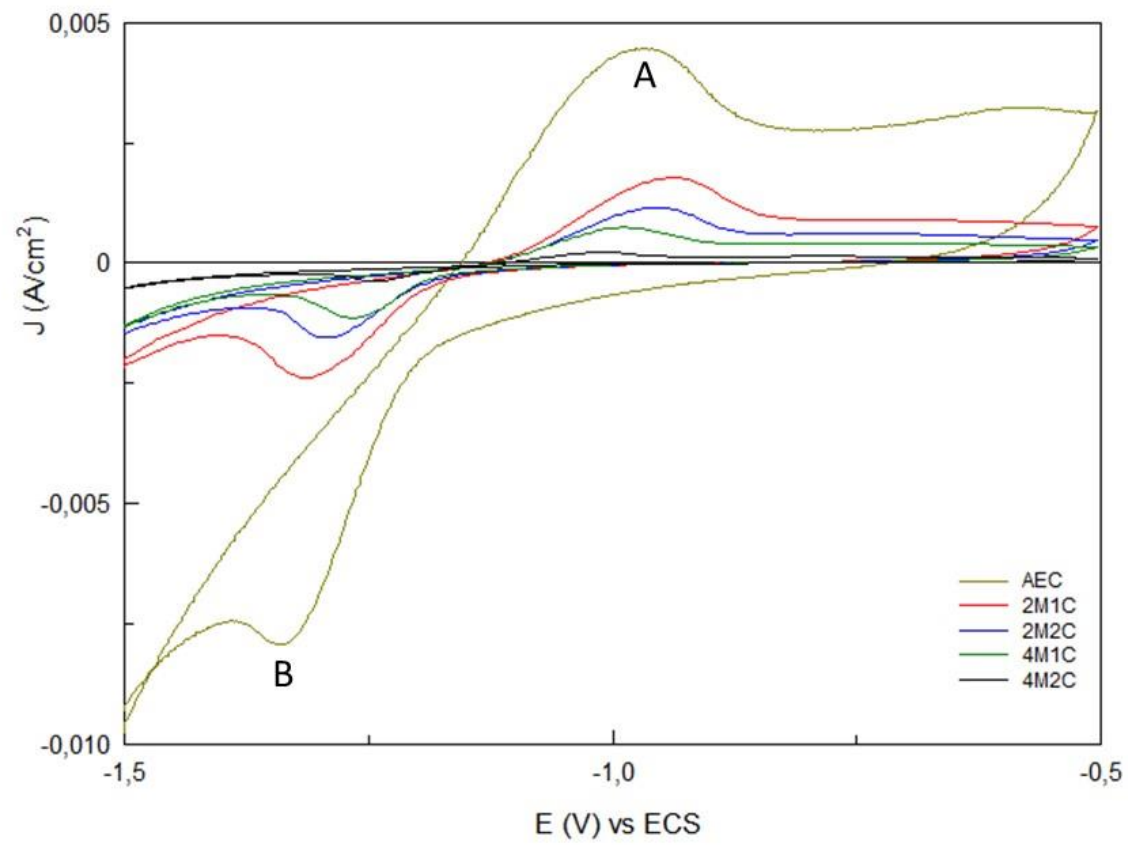

Figura 4: VC de las muestra ensayadas.

Los voltamperogramas correspondientes a las muestras recubiertas con MTMO (Figura 4) muestran en mayor o menor medida una disminución del área del pico anódico. En la Tabla 2 se muestran los resultados de la porosidad relativa (Pr) de cada una de ellas respecto al acero electrocincado sin recubrir (muestra AEC), que representa el 100\% del área expuesta. La muestra 2M1C fue la que presentó la mayor porosidad. Esto está de acuerdo con lo observado por SEM (Figura 3a), ya que fue el único recubrimiento que no era homogéneo. La segunda inmersión (muestra 2M2C) si bien produce un aumento del espesor de la película de aproximadamente el 10\%, la porosidad se reduce casi a la mitad. Esto puede relacionarse a que en la segunda inmersión el silano penetra en los poros de la película inicialmente formada sobre el cinc desnudo. El efecto de duplicar la concentración y realizar dos inmersiones (muestra 4M2C) produce un incremento de espesor del $26 \%$ y reduce la porosidad en un orden de magnitud respecto a la muestra 2M1C. Esta misma tendencia puede observarse en el ensayo de porosidad realizado con sulfato de cobre (Figura 5), en el cual la mayor reacción se produjo en la muestra AEC seguida por la 2M1C, las muestras 2M2C y 4M1C tuvieron un nivel de reacción muy similar y la que presento el mejor comportamiento, coincidente con la porosidad determinada en el ensayo de VC fue la 4M2C.

\subsection{Comportamiento frente a la corrosión}

Las curvas potenciodinámicas (Figura 6) mostraron que todas las muestras recubiertas presentaron una densidad de corriente de corrosión $\left(\mathrm{j}_{\text {corr }}\right.$ ) inferior a la del acero electrocincado. Si se compara la performance de los distintos recubrimientos, el comportamiento más pobre fue presentado por la muestra $2 \mathrm{M} 1 \mathrm{C}$, las $2 \mathrm{M} 2 \mathrm{C}$ y la $4 \mathrm{M} 1 \mathrm{C}$ tuvieron comportamientos intermedios y la mejor performance fue la de la muestra 4M2C, para la cual la $\mathrm{j}_{\text {corr }}$ disminuyó más de un orden de magnitud respecto a la de la muestra de acero electrocincado des- 
nudo (AEC).

Tabla 2: Área anódica (Oa) y porosidad relativa (Pr) de los recubrimientos de MTMO determinados por VC.

\begin{tabular}{l|l|l|l|l|}
\hline MUESTRA & Qa & Unidad & Pr & Unidad \\
\hline 2M1C & 3,00 & $\mathrm{mC} / \mathrm{cm}^{2}$ & 27,52 & $\%$ \\
\hline 2M2C & 1,70 & $\mathrm{mC} / \mathrm{cm}^{2}$ & 15,60 & $\%$ \\
\hline 4M1C & 1,30 & $\mathrm{mC} / \mathrm{cm}^{2}$ & 11,93 & $\%$ \\
\hline 4M2C & 0,24 & $\mathrm{mC} / \mathrm{cm}^{2}$ & 2,20 & $\%$ \\
\hline AEC & 10,90 & $\mathrm{mC} / \mathrm{cm}^{2}$ & 100,00 & $\%$ \\
\hline
\end{tabular}

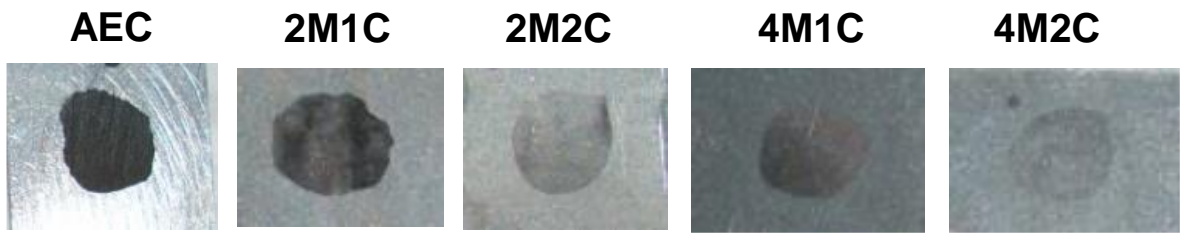

Figura 5: Fotografía de las muestras luego de aplicar una gota de solución de sulfato de cobre y esperar $10 \mathrm{~s}$.

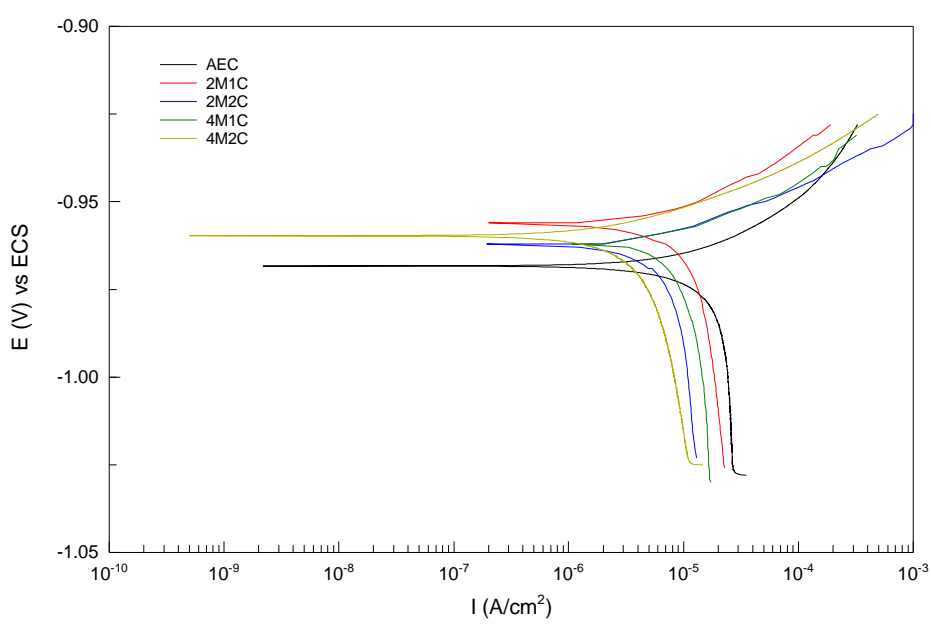

\begin{tabular}{|c|c|}
\hline Muestra & $\left(\mathrm{j}_{\text {corr }}\right)_{\mathrm{i}} /\left(\mathrm{j}_{\text {corr }}\right)_{\mathrm{AEC}}$ \\
\hline $\mathrm{AEC}$ & 1 \\
\hline 2M1C & 0,34 \\
\hline $2 \mathrm{M} 2 \mathrm{C}$ & 0,15 \\
\hline 4M1C & 0,25 \\
\hline 4M2C & 0,05 \\
\hline
\end{tabular}

Figura 6: CP y densidad de corriente de corrosión de las muestras ensayadas.

Los barridos de impedancia presentados en la Figura 7 muestran que a muy cortos tiempos de inmersión, en general, se desarrolla un solo semicírculo observándose para la muestra 4M2C otra contribución a frecuencias más bajas. A tiempos mayores, en todos los casos se define un segundo semicírculo que puede ser vinculado a la respuesta del sustrato base.

Del análisis de los resultados obtenidos a los largo de las $24 \mathrm{~h}$ de inmersión puede observarse que, independientemente de la concentración de MTMO las probetas que consideraban el recubrimiento aplicado en dos capas fueron las que presentaron mayor impedancia y, por ende, mayor efecto barrera. De la comparación del comportamiento de las muestras $2 \mathrm{M} 2 \mathrm{C}$ y $4 \mathrm{M} 2 \mathrm{C}$ se observa que en la primera de ellas se produce un aumento de la impedancia con el tiempo alcanzando valores levemente mayores a los correspondientes a 4M2C, cuya impedancia no muestra cambios significativos durante la inmersión. Este comportamiento puede ser relacionado a la presencia de productos de corrosión que bloquean las posibles imperfecciones presentes en la película de MTMO. Las tendencias de comportamiento observadas por EIE resultan consistentes con los valores de velocidad de corrosión determinados a partir de los ensayos de corriente continua. 

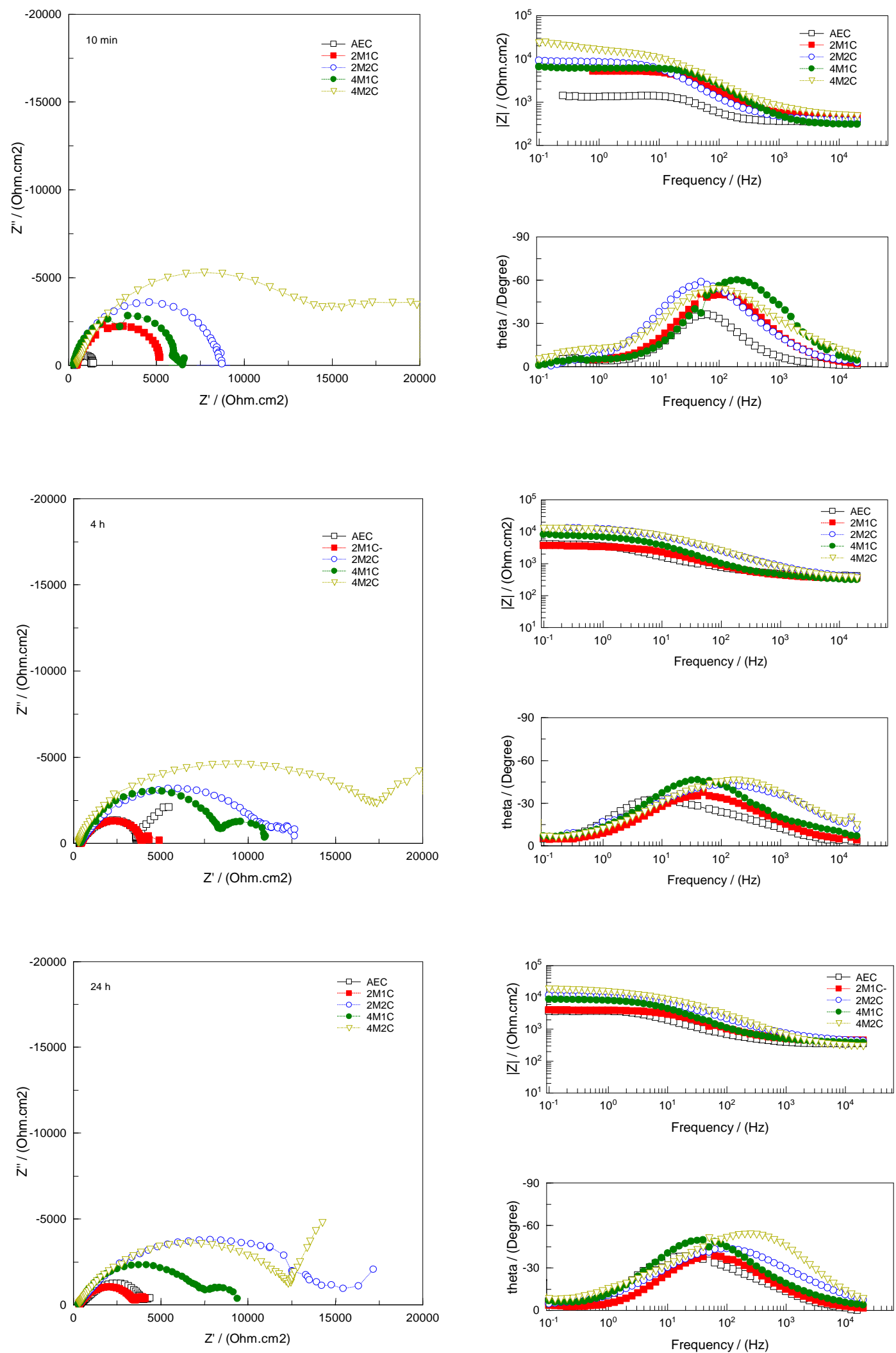

Figura 7: Diagrama de Nyquit y Bode de las muestras a distintos tiempos de inmersión en $\mathrm{NaCl}$.

Dado que el módulo de impedancia a bajas frecuencias puede ser considerado como la resistencia total del sistema [19], en la Figura 8 se presenta en forma comparativa la evolución de su valor a lo largo de la inmersión para todas las muestras consideradas. Del análisis de estos resultados puede inferirse que para el 
sustrato desnudo (AEC) la impedancia total del sistema aumenta en los primeros estadios de inmersión, debido a la formación y precipitación de productos de corrosión (óxidos e hidróxidos de cinc) y luego se estabiliza en aproximadamente $5.10^{3} \Omega . \mathrm{cm}^{2}$. En el caso de la muestra $2 \mathrm{M} 1 \mathrm{C}$, en la que se había determinado la presencia de una alta densidad de poros, la barrera aportada por la película es prácticamente despreciable y la impedancia total del sistema resulta del mismo orden que la observada para el sustrato desnudo. Por su parte, las muestras $2 \mathrm{M} 2 \mathrm{C}$ y $4 \mathrm{M} 1 \mathrm{C}$ aportan una barrera más significativa $\left(|\mathrm{Z}| \cong 6-8.10^{3} \Omega . \mathrm{cm}^{2}\right)$ al inicio de la inmersión y su magnitud aumenta hasta el final del ensayo para la muestra $2 \mathrm{M} 2 \mathrm{C}$ y para la muestra $4 \mathrm{M} 1 \mathrm{C}$ se estabiliza a partir de las $4 \mathrm{~h}$ de inmersión. Esta diferencia de comportamiento puede relacionarse con la morfología del recubrimiento de MTMO y el efecto bloqueante generado por los productos de corrosión del cinc. En consonancia con los resultados obtenidos en los restantes ensayos, la muestra 4M2C fue la que presentó desde el inicio de la inmersión una mayor impedancia total, $\left(|\mathrm{Z}| \cong 27-28.10^{3} \Omega . \mathrm{cm}^{2}\right)$, la que fue disminuyendo con el tiempo de inmersión alcanzando a las $24 \mathrm{~h}$ un valor de $15.10^{3} \Omega . \mathrm{cm}^{2}$. Este comportamiento es indicativo del más importante y efectivo efecto barrera aportado por la película generada con la mayor concentración de MTMO y aplicada en dos capas.

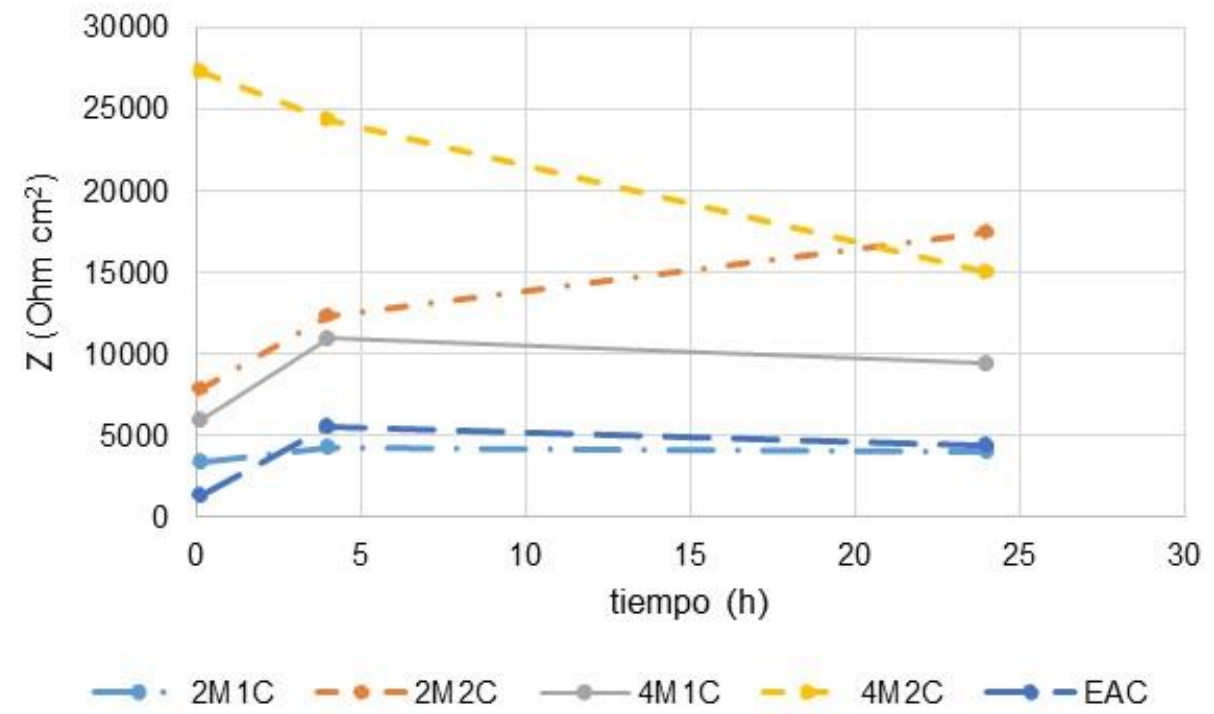

Figura 8: Evolución del módulo de la impedancia a bajas frecuencias.

En el ensayo de exposición en cámara de humedad $(\mathrm{CH})$ todos los recubrimientos mostraron un grado de protección contra la corrosión del acero electrocincado, en este caso si bien el mejor comportamiento también lo presentó la muestra 4M2C las diferencias fueron menos marcadas que en los ensayos electroquímicos.

\section{CONCLUSIONES}

Todos los recubrimientos considerados aportan algún grado de protección temporaria contra la corrosión del acero electrocincado. El aumento de la concentración de MTMO en solución produce un aumento de espesor del recubrimiento aunque dicho aumento no resulta linealmente dependiente de la concentración. Duplicar la concentración de MTMO produce un aumento en el espesor de aproximadamente el 12\%. Un efecto similar se obtiene de aplicar dos capas de MTMO en una solución de la misma concentración de silano. La aplicación de la segunda capa disminuye drásticamente la porosidad del recubrimiento con un aumento en el espesor mucho menos significativo. Esto incrementa considerablemente la resistencia frente a la corrosión del sistema. 


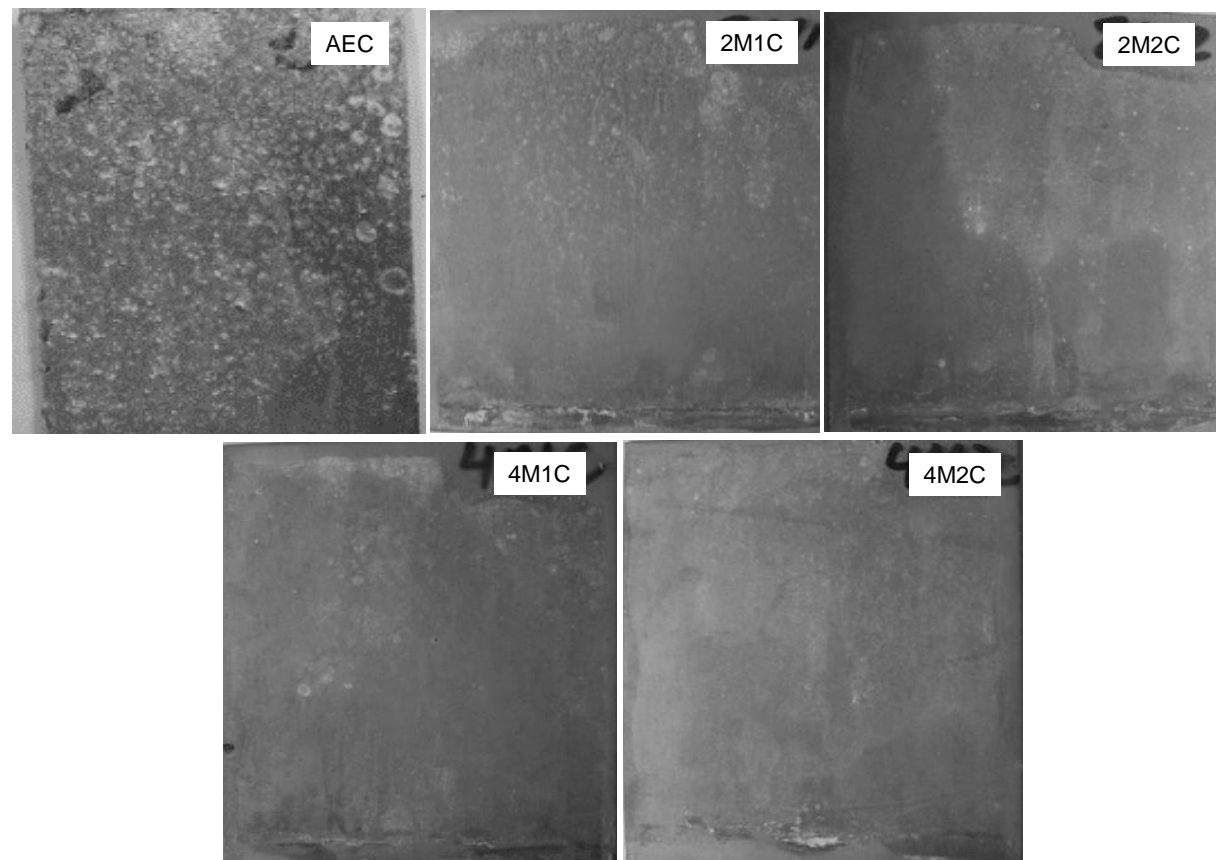

Figura 9: Fotografía de las muestras luego de 264 h en CH.

\section{AGRADECIMIENTOS}

Los autores agradecen a la Comisión de Investigaciones Científicas de la Provincia de Buenos Aires (CICPBA), al Consejo Nacional de Investigaciones Científicas y Técnicas (CONICET) y a la Universidad Nacional de La Plata (UNLP) por el apoyo económico brindado para la realización del presente trabajo.

\section{BIBLIOGRAFÍA}

[1] MARDER, A. R., "The metallurgy of zinc-coated Steel”, Progress in Materials Science, v. 45, n. 3, 191271, 2000.

[2] ASM, ASM Handbook - Corrosión, ASM International, 1992.

[3] SERVICE, U. S. P. H., “Toxicological Profile for Chromium, Agency for Toxic Substances”, In: n. ASTSDR/TP-88/10 ed., U.S. Public Health Service, 2010.

[4] VAN, O., W. J., ZHU, D., STACY, M., et al., "Corrosion Protection Properties of Organofunctional Silanes-An Overview", Tsinghua Science \& Technology, v.10, n. 6, pp. 639-664, 2005.

[5] FERREIRA, M. G. S., DUARTE, R. G., MONTEMOR, M. F., et al., "Silanes and rare earth salts as chromate replacers for pre-treatments on galvanised Steel", Electrochemical Acta., v. 49, pp. 2927-2935, 2004.

[6] NAJARI, A., LANG, P., LACAZE, P. C., et al., "Adsoption of 3-mercaptopropyltrimethoxysilane on zinc: A study of the competition between thiol and silanol functions related to the age of the siloxane solution, its pH and the oxidation state of the surface", Surface Science, v. 606, n.3-4, pp. 137-145, 2012.

[7] MAHDAVIAN, M., RAMEZANZADEH, B., AKBARIAN, M., et al., "Enhancement of silane coating protective performance by using a polydimethylsiloxane additive", Journal of Industrial and Engineering Chemistry, v. 55, n. Supplement C, pp.244-252, 2017.

[8] KUNST, S. R., MATOS, J. F., KORB, M. A., et al., "Obtención y caracterización de revestimientos protectores a base de silanos para la protección de aceros galvanizados", Información Tecnológica, v. 22, n.5, pp. 67-76, 2011.

[9] BERA, S., ROUT, T. K., UDAYABHANU, G., et al., "Water-based \& eco-friendly epoxy-silane hybrid coating for enhanced corrosion protection \& adhesion on galvanized Steel", Progress in Organic Coatings, v.101, n. Supplement C, pp. 24-44, 2016.

[10] RAMEZANZADEH, B., RAEISI, E., MAHDAVIAN, M.,"Studying various mixtures of 3aminopropyltriethoxysilane (APS) and tetraethylorthosilicate (TEOS) silanes on the corrosion resistance of 
mild steel and adhesion properties of epoxy coating", International Journal of Adhesion and Adhesives, v. 63, n. Supplement C, 166-176, 2015.

[11] DAVE, B. C., HU, X. K., DEVARAJ, Y., et al., "Sol-Gel-Derived Corrosion-Protection Coatings", Journal of Sol-Gel Science and Technology”, v. 32, n.1-3, pp. 143-147, 2004.

[12] ONO, S., TSUGE, H., NISHI, Y., et al., "Improvement of corrosion resistance of metals by an environmentally friendly silica coating method", Journal of Sol-Gel Science and Technology, v.29, n.3, pp. 147-153, 2004.

[13] BEXELL, U., GREHK, T. M., “A corrosion study of hot-dip galvanized steel sheet pre-treated with $\gamma$ mercaptopropyltrimethoxysilane”, Surface and Coatings Technology, v. 201, n. 8, pp.4734-4742, 2007.

[14] TITZ, T., HÖRZENBERGER, F., VAN DEN BERGH, K. et al., "Correlation of interfacial electrode potential and corrosion resistance of plasma polymer coated galvanized steel. Part 2: Influence of forming induced defects", Corrosion Science, v. 52, n. 2, pp.378-386, 2010.

[15] SERÉ, P. R., DEYÁ, C., EGLI, W. A., et al., "Protection of Galvanized Steel with Silanes: Its Comparison with Chromium(VI)", Journal of Materials Engineering and Performance, v. 23, pp.378-386, 2014.

[16] SERÉ, P. R., BANERA, M., EGLI, W. A., et al., "Effect on temporary protection and adhesion promoter of silane nanofilms applied on electro-galvanized Steel", International Journal of Adhesion and Adhesives., 65, n. Supplement C, pp.88-95, 2016.

[17] DATTILO, M., "Polarization and Corrosion of Electrogalvanized Steel-Evaluation of Zinc Coatings Obtained from Waste - Derived Zinc Electrolytes", Journal of the Electrochemical Society, v.132, pp. 25572561, 1985.

[18] HANSAL, W. E. G., HANSAL, S., PÖLZLER, M., et al., "Investigation of polysiloxane coatings as corrosion inhibitors of zinc surfaces”, Surface and Coatings Technology., v. 200, n. 9, pp.3056-3063, 2006.

[19] BARCELO, G., SARRET, M., MÜLLER, C., "Corrosion Resistance and Mechanical Properties of Zinc Electrocoatings”, Electrochimica Acta., v. 43,v.1-2, pp. 13-20, 1998. 